CHROMOSOME BIOLOGY

\title{
A stairway to mitotic chromosome assembly
}

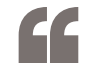

metaphase chromosomes are formed of nested loops
Upon entry into mitosis, loosely packed chromatin undergoes extensive compaction to form condensed chromosomes, which is essential for accurate segregation of genetic material and normal cell division. Over the past century, several models have been proposed as to how mitotic chromosomes form. Collaborative work from the Mirny, Earnshaw and Dekker laboratories, published in Science, now sheds light on this fundamental process, revealing that mitotic chromosomes are formed of nested loops that are tightly packed along a central helical scaffold.

To study how the morphology of mitotic chromosomes arises, the authors modified a chicken DT40 cell line to trigger a synchronous entry into mitosis and investigated chromatin organization genome-wide using $\mathrm{Hi}-\mathrm{C}$ at different time points after induction of mitotic entry. Previous models compared the organization of mitotic chromosomes to a bottlebrush, in which an array of loops emanates from a central scaffold. Using coarse-grained models and

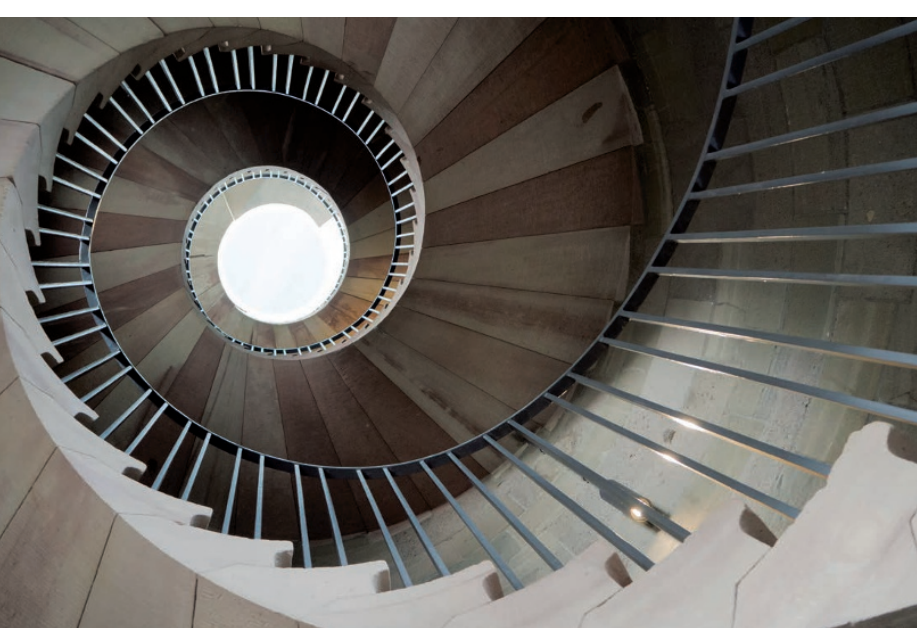

Getty/Benjamin Egerland/EyeEm by fitting model parameters to the $\mathrm{Hi}-\mathrm{C}$ data, the authors confirmed this bottlebrush-like organization, which was evident already in prophase. Notably, Hi-C data also revealed that the patterns of long-range 3D chromatin interactions characteristic of interphase cells, including formation of topologically associated domains and interactions between distal transcriptionally active or between distal transcriptionally inactive chromatin compartments, were lost within $10 \mathrm{~min}$ after entering mitosis (late prophase).

Importantly, starting from 15 min after triggering mitosis, Hi-C detected the appearance of distinct, long-range 3D interactions in chromatin, which were not observed in interphase. These interactions were observed in all mitotic chromosomes and occurred all along the chromosomes between regions separated by $\sim 3 \mathrm{Mb}$ at $15 \mathrm{~min}$ (prometaphase) and $\sim 12 \mathrm{Mb}$ at $60 \mathrm{~min}$ (metaphase). This suggested that condensing chromosomes have a periodic organization on the megabase scale. This periodicity could reflect the organization of mitotic chromatin into a helical coil. Such helical arrangement had been observed in chromosome preparations from early studies, but it was unclear whether the entire chromosome is folded to form a solenoid (external helix or solenoid model) or whether the consecutive loops wind around a central helix (internal helix or staircase model). Coarse-grained models favoured the latter arrangement. Detailed polymer simulations enabled exploration of the architecture of condensing chromosomes in more detail, showing that the pitch of the central helix progressively increases over time, which supports gradual chromosome compaction and that the higher density of chromatin observed during progressive compaction could only be achieved if metaphase chromosomes are formed of nested loops, whereby larger loops of $\sim 400 \mathrm{~kb}$ emanate from the central axis and are subdivided into smaller loops of $\sim 80 \mathrm{~kb}$.

Condensin I and condensin II complexes have previously been associated with mitotic chromosome condensation. Depletion of both condensins prevented formation of well-resolved, rod-shaped chromatids and compromised mitotic progression, resulting in the formation of tetraploid cells. Hi-C experiments followed by polymer simulations in cells lacking either condensin I or condensin II indicated that condensin II is necessary for the formation of the larger loops and their helical arrangement, whereas condensin I drives the formation of the smaller, nested loops. Notably, depletion of both condensins did not inhibit chromatin condensation, but it led to the retention of interphase $3 \mathrm{D}$ interactions within mitotic chromatin. Thus, chromatin condensation and condensin-driven establishment of mitotic chromatin organization and chromosome morphology are two separate processes.

In summary, this study uncovered the principles of how well-defined chromosomes are assembled during mitosis, at the same time uniting several previous observations and models of this process. These findings provide a solid base for studying the molecular mechanisms governing mitotic chromatin organization and chromosome morphology.

Paulina Strzyz

ORIGINAL ARTICLE Gibcus J. H., Samejima K., Goloborodko A. et al. A pathway for mitotic chromosome formation. Science https://doi. org/10.1126/science.aao6135 (2018) FURTHER READING Schmitt, A. D., Hu, M. \& Ren, B. Genome-wide mapping and analysis of chromosome architecture. Nat. Rev. Mol. Cell Biol. 17, 743-755 (2016) 